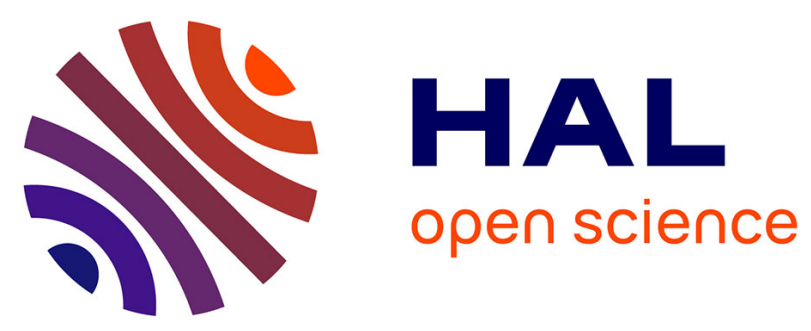

\title{
Résonance paramagnétique de défauts générés par irradiation neutronique dans l'arséniure de gallium
}

A. Goltzene, B. Meyer, C. Schwab

\section{To cite this version:}

A. Goltzene, B. Meyer, C. Schwab. Résonance paramagnétique de défauts générés par irradiation neutronique dans l'arséniure de gallium. Revue de Physique Appliquée, 1983, 18 (11), pp.703-707. 10.1051/rphysap:019830018011070300 . jpa-00245134

HAL Id: jpa-00245134

https://hal.science/jpa-00245134

Submitted on 1 Jan 1983

HAL is a multi-disciplinary open access archive for the deposit and dissemination of scientific research documents, whether they are published or not. The documents may come from teaching and research institutions in France or abroad, or from public or private research centers.
L'archive ouverte pluridisciplinaire HAL, est destinée au dépôt et à la diffusion de documents scientifiques de niveau recherche, publiés ou non, émanant des établissements d'enseignement et de recherche français ou étrangers, des laboratoires publics ou privés. 
Classification

Physics Abstracts

$61.70 \mathrm{Eg}-61.80 \mathrm{Hg}-76.30 \mathrm{Mi}$

\title{
Résonance paramagnétique de défauts générés par irradiation neutronique dans l'arséniure de gallium
}

\author{
A. Goltzene, .B. Meyer et C. Schwab \\ Laboratoire de Spectroscopie et d'Optique du Corps Solide $\left(^{*}\right)$, Université Louis Pasteur, \\ 5 , rue de l'Université, 67000 Strasbourg, France
}

(Reçu le 25 juillet 1983, accepté le 1er août 1983)

\begin{abstract}
Résumé. - Nous avons utilisé les spectres de résonance paramagnétique obtenus entre 4,2 et $300 \mathrm{~K}$ sur GaAs après irradiation aux neutrons rapides, en décomposant le spectre expérimental en un quadruplet contraint aux paramètres du modèle $\mathrm{As}_{\mathrm{Ga}}^{4+}$ et un singulet. La variation de la constante hyperfine du quadruplet et celle de l'intensité, anormale par rapport à une loi de Curie, ont été attribuées à un effet d'échange entre $\mathrm{As}_{\mathrm{Ga}}^{4+}$ et un défaut associé, responsable du singulet. Le rendement de création de ce complexe est de $10^{2}$ par neutron rapide absorbé.
\end{abstract}

\begin{abstract}
Electron paramagnetic spectra of fast neutron irradiated GaAs, obtained in the 4.2-300 $\mathrm{K}$ temperature range, have been analysed in terms of a quadruplet, whose parameters are constrained to the $\mathrm{As}_{\mathrm{Ga}}^{4+}$ model, and a singlet. The temperature variation of the quadruplet hyperfine constant and the intensity, which does not follow a Curie law, are ascribed to an exchange interaction between $\mathrm{As}_{\mathrm{Ga}}^{4+}$ and an associated defect, like related to the singlet line. The generation rate corresponds to some $10^{2}$ complex defects per absorbed fast neutron.
\end{abstract}

\section{Introduction.}

Des progrès récents dans l'élaboration de l'arséniure de gallium monocristallin et massif permettent dorénavant de produire ce dernier directement sous forme semi-isolante sans devoir procéder au dopage compensateur habituel [1-3]. Ce succès dans l'élimination des contaminants extrinsèques risque maintenant de donner un rôle accru aux défauts intrinsèques dans ce type de matériau.

Par ailleurs, les mesures de transport électriques indiquent que le niveau donneur profond EL2, non identifié à ce jour quant à sa nature physico-chimique, subsiste toujours comme défaut résiduel majoritaire $[4,5]$. Cette persistance d'un même défaut dans des matériaux, qui ont en commun une élaboration à partir du bain fondu, pourrait en suggérer une origine intrinsèque.

Enfin, il convient de se rappeler que la technique du dopage par implantation ionique comporte une phase initiale où sont générés de nombreux défauts intrinsèques, liés à l'amorphisation du réseau cristallin, lesquels devront être éliminés au cours de la phase suivante de recuit où s'effectue leur guérison et l'activation électrique des ions implantés.

Ce contexte général justifie d'accorder une impor-

(*) Laboratoire associé au CNRS, no 232. tance particulière à l'identification des défauts de nature intrinsèque dans $\mathrm{GaAs}$, d'autant plus que dans ce matériau seul l'antisite anionique $\mathrm{As}_{\mathrm{Ga}}$ a été clairement identifié en termes de configuration électronique [6].

Le recours à des faisceaux de particules élémentaires est une méthode de choix pour la création de défauts dans une matrice solide [7]. D'une manière générale, pour les semiconducteurs la plupart des études ont fait appel à une irradiation par un faisceau d'électrons de haute énergie $(\sim 1 \mathrm{MeV})$, alors que pour les métaux et alliages l'intérêt s'est concentré sur les irradiations par neutrons [8].

Ces dernières peuvent également être utilisées pour les semiconducteurs avec l'avantage supplémentaire que les conditions de création des défauts sont alors beaucoup plus proches de celles rencontrées lors d'une implantation ionique. En effet, si l'énergie d'un électron d'un MeV lui permet de déplacer un ou deux atomes dans le réseau, un neutron de même énergie donnera lieu à une cascade de déplacements d'une centaine d'atomes environ.

On sait en outre que l'antisite anionique $\mathrm{As}_{\mathrm{Ga}}$, identifié par résonance paramagnétique électronique (RPE) submillimétrique (337 GHz) sur du matériau brut de croissance [6] peut aussi être créé par irradiation aux neutrons rapides à des concentrations suffisantes de sorte à en permettre l'observation à la fréquence de $9 \mathrm{GHz}$ [9-11]. 
Ces spectres sur antisites artificiels soulèvent quelques questions d'ordre fondamental dans la mesure où les taux de génération d'As $s_{\mathrm{Ga}}$ par neutron incident semblent anormalement faibles $[9,10]$ et où l'une des composantes du quadruplet semble avoir systématiquement une intensité et une largeur supérieures à celles des autres [9-11]. En effet, l'analyse théorique attribue la signature de $\mathrm{As}_{\mathrm{Ga}_{\mathrm{a}}}$ à un spectre de structure hyperfine, correspondant au modèle d'un ion $\mathrm{As}^{4+}$. $(S=1 / 2, \quad I=3 / 2)$ entouré de quatre ions $\mathrm{As}^{3-}$ équivalents. Ce dernier donne lieu à un quadruplet formé de raies d'égale amplitude telle qu'il est effectivement observé à $337 \mathrm{GHz}$. Il s'y ajoute, entre 4,2 et $300 \mathrm{~K}$, un rapport anormal des intensités par rapport à la loi de Curie et un rétrécissement de la séparation hyperfine [11], similaire à celui observé sur $\mathrm{P}_{\mathrm{Ga}}^{4+}$ dans $\mathrm{GaP}$ [12].

Dans le but d'éclaircir ces différents points, nous avons mesuré sur les spectres RPE relevés à $4,2 \mathrm{~K}$ le taux de génération de $\mathrm{As}_{\mathrm{Ga}}$ sous irradiation aux neutrons rapides pour des fluences s'étageant de $10^{15}$ à $10^{17} \mathrm{~cm}^{-2}$, en décomposant le spectre expérimental en un quadruplet contraint aux paramètres du modèle théorique et en un singulet. Enfin, nous avons repris cette même décomposition pour analyser le spectre obtenu entre 4,2 et $300 \mathrm{~K}$ pour un échantillon irradié.

\section{Partie expérimentale.}

Le matériau était du GaAs semi-isolant d'origine commerciale (Metal Research), préparé par tirage Czochralski sous encapsulation liquide.

Les irradiations ont été effectuées sous un flux de neutrons rapides de $7 \times 10^{10} \mathrm{~cm}^{-2} \mathrm{~s}^{-1}$, la température locale ne dépassant pas $40^{\circ} \mathrm{C}$. Les neutrons thermiques sont éliminés par un blindage de $2 \mathrm{~mm}$ de $\mathrm{Cd}$ métallique. Dans ces conditions, l'énergie moyenne d'un neutron est de $2 \mathrm{MeV}$, avec un pic de distribution vers $0,8 \mathrm{MeV}$.

Les mesures de RPE ont été effectuées à $9 \mathrm{GHz}$, un même standard NBS servant de référence pour la détermination des intensités et pour la calibration des températures.

La décomposition du spectre expérimental s'est faite sur la base d'un diagramme d'énergie, dérivé de la formule de Breit-Rabi convolué par une gaussienne pour le quadruplet et d'une fonction lorentzienne pour le singulet, un polynôme d'ordre 3 traduisant la variation de la ligne de base.

\section{Résultats.}

La figure 1 montre un spectre typique, relevé à $4,2 \mathrm{~K}$, d'un échantillon irradié, la courbe inférieure traduisant les écarts entre les points expérimentaux et la courbe ajustée. Le résultat de la décomposition correspondante en quadruplet de gaussiennes et singulet lorentzien est donné par la figure 2 , après une première intégration.

La figure 3 représente la variation du nombre de

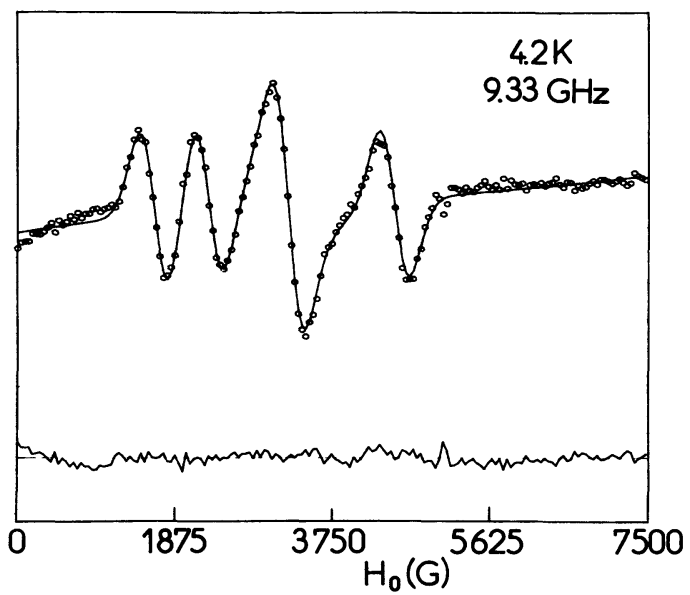

Fig. 1. - Spectre RPE de GaAs semi-isolant non dopé irradié aux neutrons rapides. La courbe continue représente la superposition d'un quadruplet de gaussienne et d'un singulet lorentzien ajustés aux données expérimentales (cercles). La courbe du bas indique les écarts entre les points expérimentaux et la courbe ajustée.

[ESR spectrum of fast neutron irradiated semi-insulating undoped $\mathrm{GaAs}$. Solid line represents the superposition of a quadruplet of Gaussian lines and a singlet Lorentzian line fitted to the data (circles). The lower curve indicates the deviation between experimental data and the best fit.]

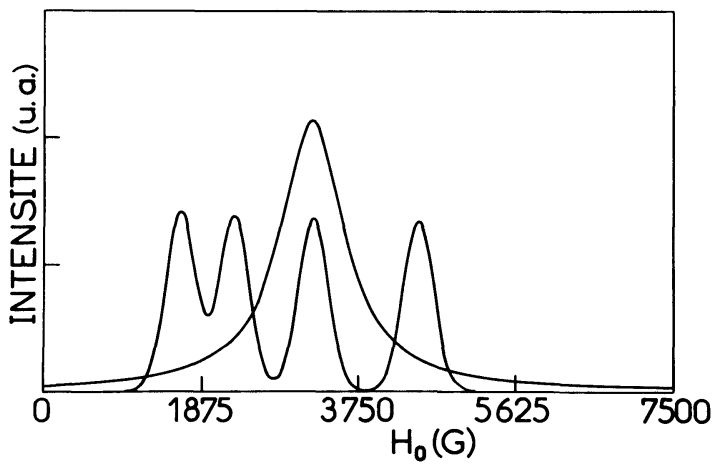

Fig. 2. - Résultat de la décomposition du spectre RPE en quadruplet de gaussienne et singulet lorentzien, après une première intégration.

[Result of the ESR spectrum decomposition by a quadruplet of Gaussian lines and a singlet Lorentzian line after integration.]

spins pour le quadruplet (nombre effectif de centres $\mathrm{As}_{\mathrm{Ga}}$ ) et pour le singulet en fonction de la fluence de neutrons rapides. La pente étant proche de l'unité, on peut en déduire un taux de génération de $115 \pm 25$ antisites par neutron absorbé.

Sur la figure 4 est portée la corrélation entre les concentrations de spins correspondant au quadruplet et celles correspondant au singulet. En l'absence d'un modèle pour le centre correspondant, seule une valeur proportionnelle à la concentration réelle peut être indiquée. La pente étant à nouveau de l'ordre de l'unité, on peut en déduire toutefois un rapport 


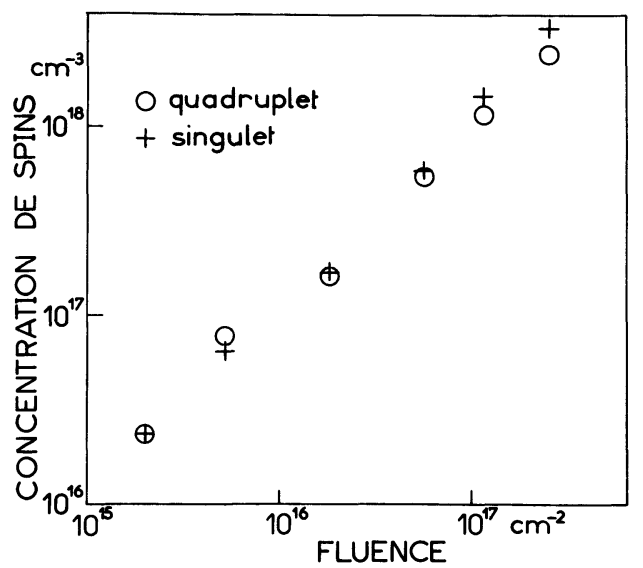

Fig. 3. - Variation de la concentration de spins pour le quadruplet $\left(=" \mathrm{As}_{\mathrm{Ga}} »\right)$ et le singulet correspondant en fonction de la fluence de neutrons rapides.

[Dependence of the spin concentration of the quadruplet (= "As $s_{\mathrm{Ga}}$ ") and the related singlet on the fast neutron fluence.]

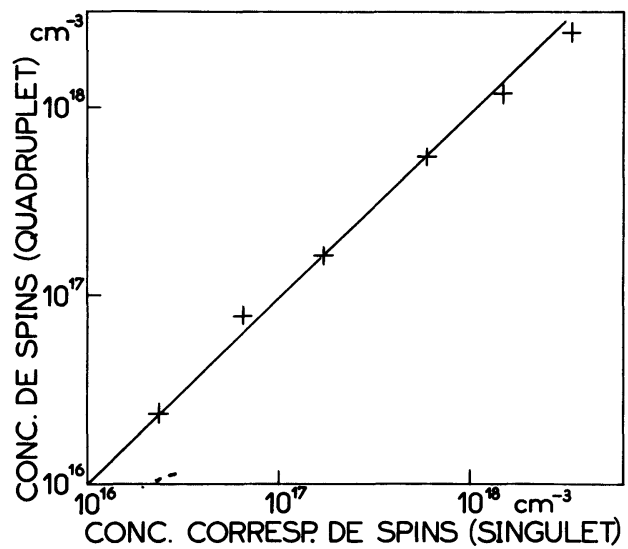

Fig. 4. - Corrélation entre les concentrations de spins correspondant au quadruplet (= "As $\mathrm{Aa}_{\mathrm{Ga}}$ ) et au singulet.

[Correlation between spin concentration of the quadruplet $\left(=\left\|\mathrm{As}_{\mathrm{Ga}}\right\|\right)$ and spin concentration of related singlet.]

constant dans le taux de génération des deux défauts correspondants.

Les figures 5 et 6 indiquent les variations de l'inverse de la susceptibilité magnétique en fonction de la température respectivement pour le quadruplet et le singulet. On notera dans les deux cas l'existence d'une région où la variation est conforme à la loi de Curie $(T<100 \mathrm{~K})$. Les points d'intersection sont obtenus à partir de droites des moindres carrés ajustées respectivement pour les points de mesure en dessous et au-dessus de $100 \mathrm{~K}$.

Enfin, la variation thermique de la constante hyperfine, traduisant la séparation entre les composantes du quadruplet de $\mathrm{As}_{\mathrm{Ga}}$ est reportée sur la figure 7. Les écarts systématiques par rapport à la droite d'ajustement suggèrent une dépendance d'ordre plus élevée en fonction de la température. De fait, un

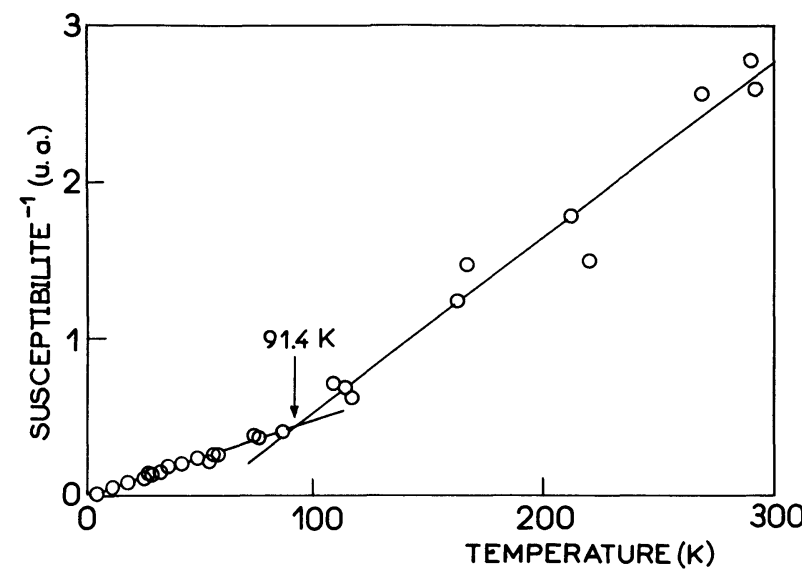

Fig. 5. - Variation de l'inverse de la susceptibilité magnétique, correspondant au quadruplet ( $=" \mathrm{As}_{\mathrm{Ga}}$ ") en fonction de la température.

[Temperature dependence of the inverse of the magnetic susceptibility of the quadruplet $\left(=" \mathrm{As}_{\mathrm{Ga}} »\right)$.]

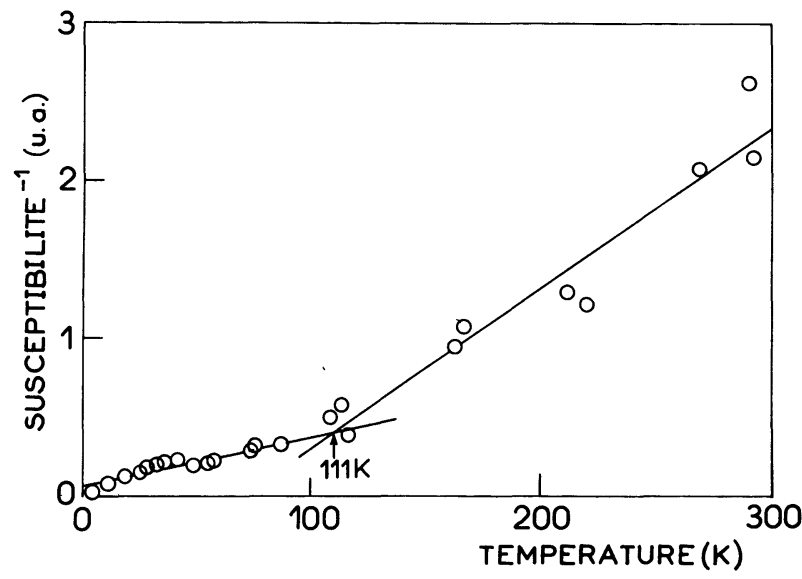

Fig. 6. - Variation de l'inverse de la susceptibilité magnétique, correspondant au singulet, en fonction de la température.

[Temperature dependence of the inverse of the magnetic susceptibility of the singlet.]

ajustement polynomial d'ordre 4 fournit un point d'inflexion à $87 \mathrm{~K}$, valeur proche des intersections relevées sur les courbes de susceptibilité.

\section{Discussion.}

Nos résultats indiquent un taux de génération des antisites sous irradiation de neutrons rapides environ dix fois supérieur à celui reporté par d'autres auteurs $[9,10]$. Cet important écart trouve certainement son origine dans les conditions différentes de l'irradiation, à savoir emploi d'un faible flux dans notre cas assurant une faible élévation de température au-dessus de l'ambiante $\left(T<40^{\circ} \mathrm{C}\right)$ par rapport à un flux intense $\left(\times 10^{3}\right)$ où la dissipation d'énergie in situ pendant l'irradiation n'est plus négligeable. Ceci est 


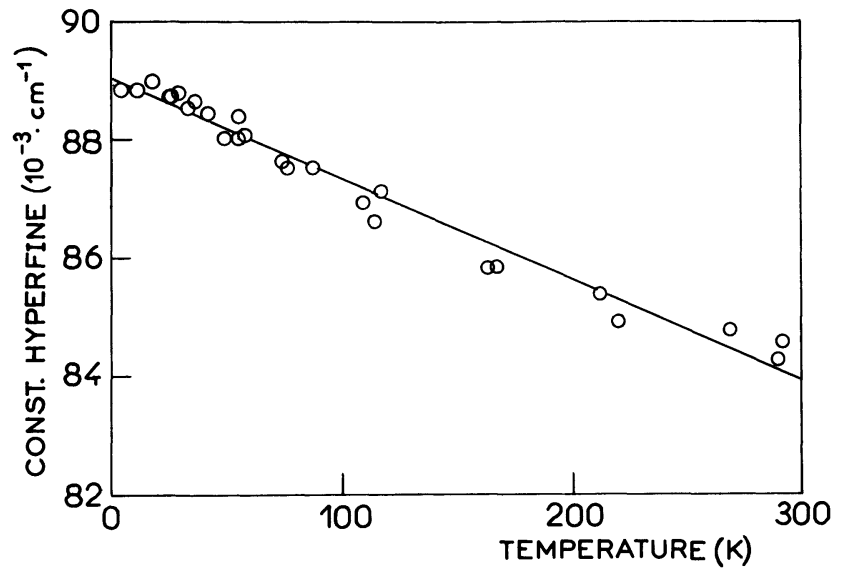

Fig. 7. - Variation thermique de la constante hyperfine du quadruplet.

[Temperature dependence of the quadruplet hyperfine constant.]

d'autant plus important qu'il existe une étape de guérison thermique des dommages d'irradiation au voisinage de $300 \mathrm{~K}$ [13], de sorte que la détermination exacte de la température de l'échantillon est un facteur important.

La déviation notable par rapport à la loi de Curie aux températures supérieures à $100 \mathrm{~K}$ pourrait en principe avoir son origine soit dans une vraie diminution du nombre des centres isolés $\mathrm{As}_{\mathrm{Ga}}$ soit dans un effet d'échange.

Dans un semiconducteur compensé, donneurs et accepteurs sont ionisés à toute température conduisant à rejeter l'hypothèse d'une diminution de la concentration des antisites par transfert d'électron sur un autre piège. Par ailleurs, l'expérience montre que les courbes de susceptibilité ne présentent pas d'hystérésis.

Un effet d'échange, tel qu'un couplage antiferromagnétique entre l'électron célibataire dans un état $S$ de l'ion $\mathrm{As}^{4+}$ et un second défaut paramagnétique proche pourrait simuler une diminution apparente du nombre de spins. Il reste toutefois à expliquer la contradiction posée par la diminution du nombre de spins isolés lors de l'augmentation de la température. Ceci n'est possible que dans la mesure où le découplage des spins résultant de l'agitation thermique est compensé par une augmentation du recouvrement des fonctions d'onde en fonction de la température.
Nous pensons que tel est le cas et donc que nous sommes en présence d'un défaut associé.

L'importante variation de la constante hyperfine avec la température qui traduit l'interaction entre la fonction électronique centrée sur le défaut et les noyaux environnants, indique qu'il se produit une forte relaxation du réseau lorsque la température croît. En principe, un tel effet pourrait se produire à l'occasion d'un effet Jahn-Teller.

Cependant, en raison de la symétrie $S$ de l'électron célibataire, un tel effet n'est pas permis sur le centre $\mathrm{As}_{\mathrm{Ga}}$ lui-même, de sorte que nous sommes amenés à conclure qu'il se produit sur le centre associé invoqué plus haut et que nous en voyons simplement l'effet indirect sur l'antisite.

Dans ce contexte, il nous semble vraisemblable que ce centre associé soit celui qui corresponde au singulet; en effet, il présente les conditions requises d'une anomalie de comportement au voisinage de la même température et d'une concentration directement corrélée à celle de $\mathrm{As}_{\mathrm{Ga}}$.

En résumé, nous avons montré qu'un second défaut était corrélé de manière quantitative à la génération des antisites anioniques $\mathrm{As}_{\mathrm{Ga}}$ par irradiation aux neutrons rapides et que la valeur numérique de $115 \pm 25$ que nous avons trouvée est plus en accord avec les prévisions [8].

La variation de l'intensité du spectre en fonction de la température semble exclure qu'il puisse s'agir d'un défaut isolé en raison d'une brutale atténuation se produisant aux températures supérieures à $100 \mathrm{~K}$. Le comportement en tout point similaire du second défaut, dont la signature RPE se limite à un profil lorentzien, indique que ce dernier, remplit les conditions nécessaires pour justifier d'une relaxation JahnTeller avec des concentrations similaires à celle de $\mathrm{As}_{\mathrm{Ga}}$, pour être le partenaire recherché dans le cadre d'un modèle de défaut associé pour les antisites anioniques générés par irradiation. Ces résultats peuvent fournir une ouverture à certains aspects controversés sur les comportements électriques d'antisites reportés dans la littérature $[14,15]$.

\section{Remerciements.}

Nous remercions M. Stampfler pour les irradiations effectuées au Service de la Pile Universitaire au Centre de Recherches Nucléaires de Strasbourg.

\section{Bibliographie}

[1] Henry, R. L., Swiggard, E. M., Inst. Phys. Conf. Ser. B 33 (1977) 28.

[2] Thomas, R. N., Hobgood, H. M., Barrett, D. L., ELDRIDGE, G. W., dans Semi-Insulating III-V Materials (Nottingham, 1980) éd. par G. J. Rees (Shiva Publishing Ltd, Orpington) 1980 p. 76.
[3] Lifang, T., Liying, L., Weizu, T., Qitung, L., YuANXI, Z., dans Semi-Insulating III-V Materials (Evian, 1982) éd. par S. Makram-Ebeid et B. Tuck (Shiva Publishing Ltd, Nantwich (GB)) 1982, p. 248. 
[4] Martin, G. M., Farges, J. P., Jacob, G., Hallais, J. P., J. Appl. Phys. 51 (1980) 2840.

[5] Holmes, D. E., Chen, R. T., Elliott, K. R., KirkPatrick, C. G., Appl. Phys. Lett. 40 (1982) 46.

[6] Wagner, J. R., Krebs, J. J., Stauss, G. H., White, A. M., Solid State Commun. 36 (1980) 15.

[7] Corbetr, J. W., Electron Radiation Damage in Semiconductors and Metals (Academic Press, New York) 1966.

[8] Gitrus, J., Irradiation Effects in Crystalline Solids (Applied Science Publishers Ltd, London) 1978.

[9] Goswami, N. K., Newman, R. C., Whitehouse, J. E., Solid State Commun. 40 (1981) 473.
[10] Worner, R., Kaufmann, U., Schneider, J., Appl. Phys. Lett. 40 (1982) 141.

[11] Goltzene, A., Meyer, B., Schwab, C., J. Appl. Phys. 54 (1983) 3117.

[12] HausmanN, A., Schamberg, F. J., dans Verhandlungen der Deutschen Gesellschaft (Munster, 1981) éd. par W. Heinicke (Physik-Verlag, Weinheim) 1981 p. 184.

[13] Wood, F. L., J. Phys. Soc. Jpn 18, suppl. II (1963) 190.

[14] Martin, G. M., Makram-Ebeid, S., dans Proc. 12th Int. Conf. on Defects in Semiconductors (Amsterdam, 1982) Physica, B 116 (1983) 371.

[15] Weber, E. R., Schneider, J., dans Proc. 12th Int. Conf. on Defects in Semiconductors (Amsterdan:, 1982). Physica, B 116 (1983) 398. 